\title{
Altered levels of the onco-microRNA 21 and the tumor-supressor microRNAs 143 and 145 in advanced rectal cancer indicate successful neoadjuvant chemoradiotherapy
}

\author{
UTA DREBBER $^{1}$, MAX LAY ${ }^{1}$, INGA WEDEMEYER ${ }^{1}$, DANIEL VALLBÖHMER ${ }^{2}$, \\ ELFRIEDE BOLLSCHWEILER ${ }^{2}$, JAN BRABENDER ${ }^{2}$, STEFAN P. MÖNIG ${ }^{2}$, \\ ARNULF H. HÖLSCHER ${ }^{2}$, HANS P. DIENES ${ }^{1}$ and MARGARETE ODENTHAL ${ }^{1}$ \\ ${ }^{1}$ Institute of Pathology; ${ }^{2}$ Department of General, Visceral and Cancer Surgery, University \\ Hospital of Cologne, Kerpener Str. 62, 50937 Cologne, Germany
}

Received February 9, 2011; Accepted April 4, 2011

DOI: 10.3892/ijo.2011.1036

\begin{abstract}
RNAs (miRNAs) are small non-coding RNAs with important post-transcriptional regulatory functions. miRNA-21 (miR-21) is upregulated and miR-143 and miR-145 are downregulated in colorectal carcinoma. The aim of our study was to determine if these miRNAs change their expression levels in response to neoadjuvant chemoradiotherapy in advanced rectal cancer. Forty patients with advanced rectal cancer (clinical uT3/T4 Nx) were included. All patients underwent neoadjuvant chemoradiotherapy and surgical resection. Expression of miR-21, -143 and -145 was examined in macrodissected tumor tissue before and after chemoradiotherapy and normal rectal tissue from the resection specimen. RNA was extracted from formalin-fixed and paraffin-embedded tissue by TRIzol method, polyadenylated, reverse transcribed and analyzed by real-time PCR. Therapy response was assessed according to pathological tumor regression. miR-21 was more highly expressed in tumor tissue than in non-tumorous tissue. However, there was a moderately lower expression in post-therapeutic tumor tissue compared to pre-therapeutic tumor tissue. There was a significant upregulation of miR-143 and miR-145 in post-therapeutic tumor tissue compared to pre-therapeutic tumor tissue. According to the predictive and prognostic value of the analyzed miRNAs, a significant correlation between miR-145 expression and tumor regression was seen. Patients with a low intratumoral post-therapeutic expression had significantly more often a worse response to neoadjuvant therapy compared to patients with a high expression of miR145. The present results support the hypothesis that chemoradiotherapy can profoundly alter miRNA expression
\end{abstract}

Correspondence to: Dr Uta Drebber, Institute of Pathology, University Hospital of Cologne, Kerpener Str. 62, 50937 Cologne, Germany

E-mail: u.drebber@uni-koeln.de

Key words: locally advanced rectal cancer, neoadjuvant therapy, response evaluation, microRNA profiles. miRNAs might play important roles as molecular biomarkers in the prediction of response to treatment and prognosis.

\section{Introduction}

Preoperative treatment of advanced rectal cancer with neoadjuvant combined chemoradiotherapy has become a widely accepted therapy strategy (1). Compared with postoperative treatment, preoperative chemoradiotherapy is associated with improved local control and reduced toxicity (2). Histopathological evaluation of tumor regression in the surgical specimen after preoperative chemoradiotherapy is believed to be an objective parameter and allows the identification of patients, who have minor or major benefit from multimodal therapy (3). However, only a subgroup of patients treated with preoperative chemoradiotherapy respond to treatment. Another subgroup are non-responders, who do not benefit and should be treated differently. Thus, one major issue is the implication of predictive molecular factors determining histopathological response to treatment (4).

microRNAs (miRNAs) are small non-coding RNAs that function as post-transcriptional regulators of gene expression in eukaryotic organisms (5-7). miRNAs have important regulatory functions in biological processes as development, cellular differentiation, proliferation and apoptosis (8). Dysregulation of miRNAs can influence carcinogenesis if their miRNA targets are encoded by tumor suppressor genes or oncogenes (9). Altered miRNA expression has been reported in various cancers, and the profiles of miRNAs exhibit great potential for an application in cancer definition (10-12). Although the precise biological role of all miRNAs has not been entirely elucidated, it has been found that each miRNA can regulate the expression of hundreds of target genes (13).

In colorectal cancer (CRC), many proteins involved in key signalling pathways are dysregulated and seem to be affected by miRNA regulation $(14,15)$. Extracellular matrix regulators and epithelial-mesenchymal transition transcription factors are also altered and affected by miRNAs. Among other miRNAs, 
miR-143 plays a key role in CRC. It has been shown that miR-143 is downregulated in CRC (15). miR-143 functions as a tumor suppressor and downregulation has a potential to drive tumor progression toward malignancy (15). Recently, KRAS oncogene has been validated as the target of miR-143 in CRC (15). It was shown, that miR-143, significantly suppresses colorectal cancer cell growth through inhibition of KRAS translation (10).

miR-145 is a miRNA which has repeatedly been reported to be downregulated in CRC and playing a pivotal role as a tumor suppressor (16-18). Findings in a study of La Rocca et al indicated that miR-145 has tumor suppressor activity which depends in large part on its ability to downregulate insulin receptor substrate-1 (IRS-1) which plays an important role in cell growth and proliferation (19).

Substantial data indicate that miR-21 which is significantly upregulated in CRC has an important function in the pathogenesis of CRC as it shows also an inverse correlation with survival (20). miR-21 strongly represses the tumor-suppressor gene PTEN which is an important regulatory component of the phosphatidylinositol-3-kinase (PI-3-K) pathway. Suppression of PTEN controlled by miR-21 is associated with augmentation of PI-3-K signalling and progression of CRC. Besides miR-21 is known to regulate multiple genes associated with cellular motility and extracellular matrix remodelling. It was demonstrated that miR-21 acts on tumor suppressor gene PDCD4 and plays a role as negative regulator of intravasation through the invasion-related urokinase receptor gene (UPAR) $(17,21,22)$.

There is evidence that some miRNAs change their levels in reaction to chemotherapeutic agents. Changes in the expression of miRNAs caused by chemotherapeutic agents such as 5-Fluorouracil (5-FU) were described using in vitro cell line systems of human colon cancer cells $(23,24)$. However, cell line-based studies do not always correlate well with results from tumor tissue and there are only few studies that have examined the effects of chemotherapeutic agents on miRNA expression in CRC in vivo $(25,26)$. Therefore, investigations on the possible influence afforded by drug treatment on miRNA expression patterns appear to be of primary interest (23).

In the present study, we compared the expression levels of miR-21, -143 and -145 in rectal cancer before and after chemoradiotherapy to detect possible therapy-induced changes in miRNA expression. We also determined whether there is an association between the modification of miRNA expression by chemoradiotherapy, tumor regression and clinical outcome.

\section{Material and methods}

Patients. Forty patients with locally advanced rectal cancer who were treated between 1999 and 2007 in the Department of General, Visceral- and Cancer Surgery of the University Hospital Cologne were included in this study. Tissue was collected from the files of the Institute of Pathology of the University of Cologne. Of the 40 patients, 24 were male and 16 were female. The median age was 60.8 years (range $35-79$ ). None of the patients received prior radiochemotherapy and/or chemotherapy. The patients with clinical advanced rectal cancer (uT3/ 4Nx) were treated with neoadjuvant chemoradio- therapy with 5-FU and 50.4 Gy followed by surgical resection. Clinical cTNM stage (preceding a therapy) was based on the endorectal ultrasonography, CT and colonoscopy. Restaging was done 2-3 weeks following completion of radiochemotherapy, and surgical therapy was performed in all study patients. The scientific protocol was approved by the local Ethics Committee.

Pathologic review of pre-therapeutic tumor biopsies. Tumor biopsies that had been obtained endoscopically during pre-therapeutic staging procedures were retrieved from the files of the Institute of Pathology at the University Hospital of Cologne. Histological slides of the biopsy specimens were stained with hematoxylin and eosin to determine tumor type and tumor grade according to WHO classification.

Histomorphologic grading of tumor regression. Residual tumors were fixed with formaldehyde (4\%) for $24 \mathrm{~h}$. The complete tumor area was embedded in paraffin blocks and investigated histologically. Serial sections were cut from each block and stained with hematoxylin and eosin. Histomorphologic assessment of tumor regression was performed by an objective histopathologic examination by a staff pathologist who was blinded for all other clinical data performed in this analysis (U.D.). The percentage of viable residual tumor cells was estimated, and patients were allocated to one of the following groups as previously described (27): grade 4, no residual tumor cells; grade $3,<10 \%$ residual tumor cells; grade 2, 10-50\% residual tumor cells; grade 1, 50-100\% residual tumor cells. For statistical analysis, the histological response was devided in major response: no residual tumor cells or $<10 \%$ of residual cells and minor response $>10 \%$ viable tumor cells.

Tissue preparation and macrodissection. From each patient formalin-fixed and paraffin-embedded samples of pretherapeutic biopsy and post-therapeutic tissue of the tumor area and of normal rectal mucosa were prepared. Six sections of $7.5 \mu \mathrm{m}$ thickness were cut, mounted on glas slides and used for macro-dissection. Macrodissection was performed as previously described (28).

RNA isolation. Formalin-fixed and paraffin-embedded samples were deparaffinized in xylene by incubation at $65^{\circ} \mathrm{C}$ for a total of $20 \mathrm{~min}$, xylene being substituted twice. After two washes with $100 \%$ ethanol, samples were lysed in $500 \mu \mathrm{g} / \mathrm{ml}$ proteinase $\mathrm{K}$ in $50 \mathrm{ml}$ TRIS, $5 \mathrm{ml}$ EDTA (Invitrogen, Karlsruhe, Germany). Total RNA was extracted by phenol/ chloroform and subsequently precipitated in $200 \mathrm{mM}$ sodium acetate and ispopropanol as described previously (28). To ensure complete removal of genomic DNA, RNA samples were treated for $30 \mathrm{~min}$ with $30 \mathrm{U}$ desoxyribonuclease (Roche, Mannheim, Germany) at $37^{\circ} \mathrm{C}$.

Reverse-transcription and real-time polymerase chain reaction $(P C R)$. Extracts of total RNA were measured with the ND-1000 NanoDrop spectrophotometer (NanoDrop, Wilmington, USA). microRNA was analyzed by a two-step real-time PCR (RT-PCR) using the miScript-reverse transcription kit and the miRNA SYBR-Green PCR kit purchased 

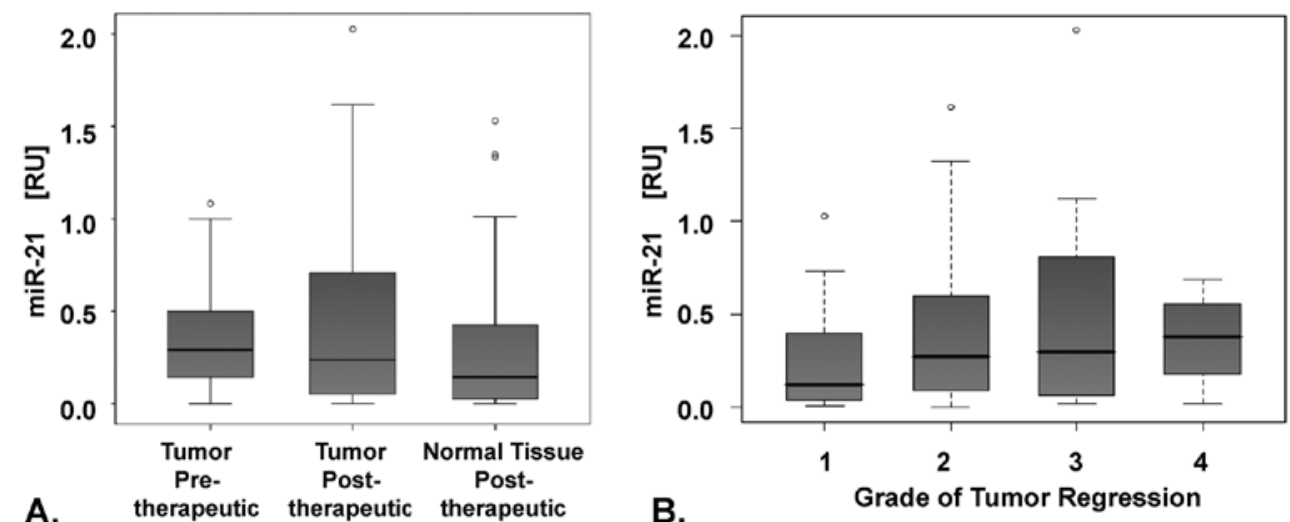

Figure 1. (A) Boxplot showing different levels of miR-21 expression. miR-21 is expressed in higher levels in pre-therapeutic tumor tissue (0.29) compared to

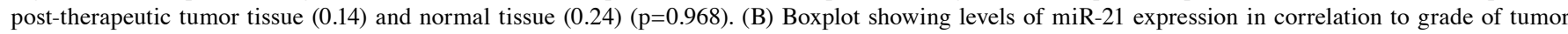
regression in post-therapeutic tumor tissue: 0.120 in grade 1 patients $(n=11), 0.270$ in grade 2 patients $(n=15), 0.29$ in grade 3 patients $(n=10), 0.378$ in grade 4 patients $(\mathrm{n}=4)(\mathrm{p}=0.657)$

from Qiagen (Hilden, Germany). The first step includes total RNA polyadenylation and reverse transcription, then followed by RT-PCR. Primers used for cDNA synthesis and RT-PCR were purchased from the GeneGlobe Search Center (Qiagen). All steps were performed in triplicates following the supplier's guidelines. Absolute mRNA quantification was carried out using a standard curve and RNU6 for normalisation.

Statistical evaluation. Data analysis: All data of miRNA were checked for normal distribution. The Friedman-test was used to determine the significance of differences between paired data sets (pre-therapeutic biopsy, tumor of the surgical specimen, normal tissue post-therapeutic). Receiver, operator curve (ROC) with $95 \%$ confidence interval was applied to find out an optimal cut-off value for prediction of response. Frequencies of categorical variables, the median, with the lower (LQ) and upper (UQ) quartiles, or minimum and maximum, were used for descriptive statistics. Differences in patient, tumor and treatment characteristics were assessed using the Mann-Whitney test for continuous variables and the Chi-square test or Fisher's exact test for categorical variables with a significance level of $\mathrm{p}<0.05$.

The median follow-up time of the surviving patients was 4.4 years (range 2.2-6.4 years). Kaplan-Meier plots were used to depict survival distribution. The log-rank test was used to evaluate for survival differences. All statistical analyses were performed using the statistic program SPSS for Windows version 18.0. For graphic presentation of the survival curves, MedCalc version 9.3 was used.

\section{Results}

Expression levels of miRNAs. In each case miRNA epression levels were analyzed in tumor tissue before and after neoadjuvant chemoradiotherapy and in normal rectal tissue of the surgical specimen after therapy.

Expression levels of $m i R-21$. The highest level of miR-21 was found in the pre-therapeutic cancer tissue of the biopsy (median 0.29 , min $0, \max 2.25$ ) followed by normal tissue (median 0.24 , min $0, \max .2 .03$ ) and post-therapeutic cancer tissue (median 0.14, min 0, max. 1.43). The differences were not statistically significant ( $\mathrm{p}=0.968$ pre-therapeutic versus posttherapeutic tumor tissue) (Fig. 1A).

Post-therapeutic expression levels of miR-21 in tumor tissue and correlation with tumor response. Compared to pretherapeutic biopsy samples miR-21 was downregulated after treatment in tumor tissue. In view of tumor regression, there was the lowest expression level of post-therapeutic intratumoral miR-21 $(0.120)$ in grade 1 patients $(n=11)$, followed by grade 2 patients $(n=15)(0.270)$, grade 3 patients $(n=10)(0.29)$ and grade 4 patients $(n=4)(0.378)$. The differences in miR-21 expression levels were not significant $(\mathrm{p}=0.657)$ (Fig. 1B).

Expression levels of $m i R-143$. The lowest level of miR-143 was found in pre-therapeutic cancer tissue (median 0.006, $\min 0$, max 0.9 ) followed by post-therapeutic cancer tissue (median 0.666, min 0.001, max 7.2) and normal tissue (median 0.666 , min 0.001, $\max 9.9$ ). The difference between pretherapeutic and post-herapeutic miR-143 levels in tumor tissue were statistically significant $(\mathrm{p}<0.0001)$ (Fig. 2A).

Post-therapeutic expression levels of miR-143 in tumor tissue and correlation with tumor response. Compared to pre-therapeutic biopsy samples miR-143 was upregulated after treatment in tumor tissue. In view of tumor regression, the epression levels of post-therapeutic intratumoral miR-143 were as follows: regression grade $1(n=11) 0.66$, regression grade 2 $(n=15) 0.35$, regression grade $3(n=10) 0.91$, and regression grade $4(n=4) 0.70$. The differences in miR-143 expression levels were not significant $(\mathrm{p}=0.895)$.

Post-therapeutic expression levels of miR-143 and correlation with overall survival. Analysis of the correlation between post-therapeutic miR-143 expression and survival showed a trend of longer survival in patients with higher miR-143 expression (cut-off value of 1). This trend was not statistically significant $(\mathrm{p}=0.231)$ (Fig. $2 \mathrm{~B})$.

Expression levels of miR-145. The lowest level of miR-145 was found in pre-treatment cancer tissue (median 0.007, 


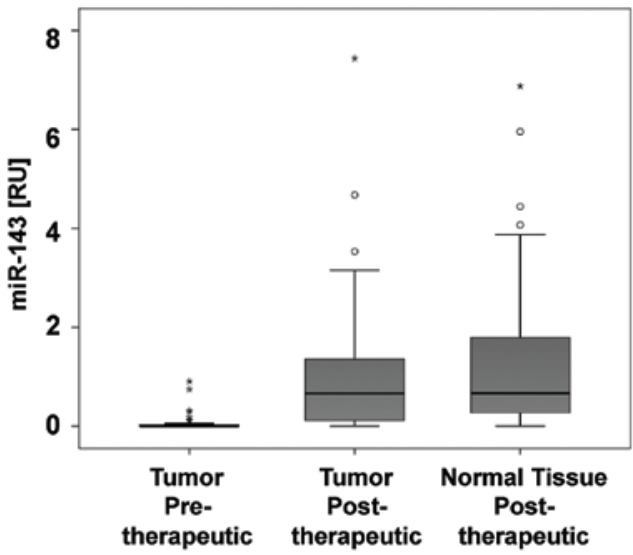

A.

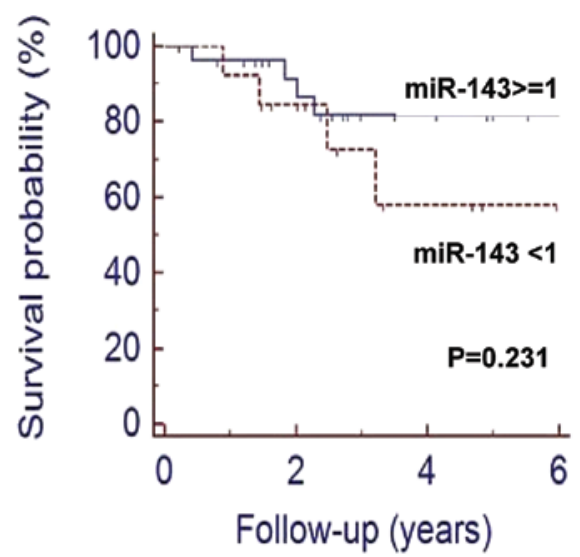

B.

Figure 2. (A) Boxplot showing the different levels of miR-143 in pre-therapeutic and post-therapeutic tumor and normal post-therapeutic tissue. There is a significant increase of miR-143 expression in post-therapeutic $(0.666)$ compared to pre-therapeutic tumor 0.006$)(\mathrm{p}<0.0001)$. (B) Kaplan-Meier overall survival curve based on post-therapeutic intratumoral miR-143 expression after neoadjuvant therapy.

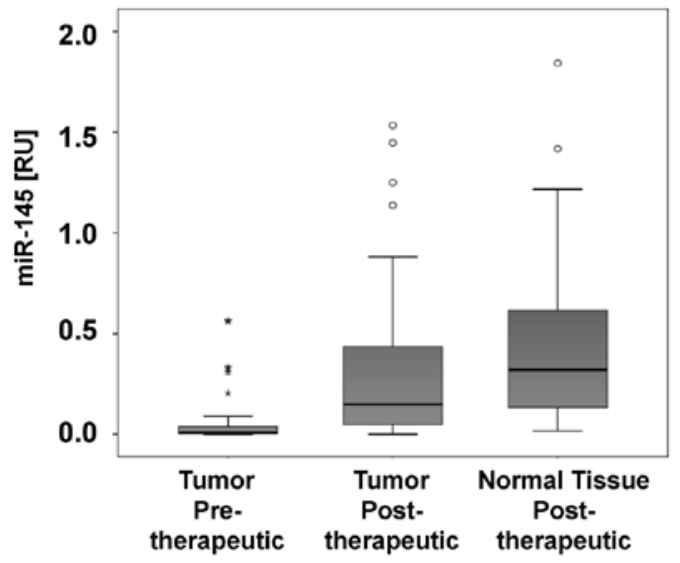

Figure 3. Boxplot showing levels of miR-145 expression in pre-therapeutic and post-therapeutic tumor tissue and normal post-therapeutic tissue. There is a significant increase of miR-143 expression in post-therapeutic $(0.147)$ compared to pre-therapeutic tumor $(0.007)(\mathrm{p}<0.001)$.

$\min 0, \max 0.564)$ followed by post-therapeutic tumor tissue (median 0.147, min 0, max 1.533) and normal post-therapeutic tissue (median 0.320 , min 0.015, $\max 7.34$ ). The difference between pre-therapeutic and post-therapeutic miR-145 levels were statistically significant $(\mathrm{p}<0.001)$ (Fig. 3).

Post-therapeutic expression levels of miR-145 in tumor tissue and correlation with tumor response. Compared to pre-therapeutic biopsy samples miR-145 was upregulated after treatment in tumor tissue. In view of tumor regression, the highest expression level of miR-145 (0.436) was found in patients with regression score $3(n=10)$ followed by patients with regression score $2(n=15)(0.264)$ and patients with regression score 4 $(n=4)(0.114)$. The lowest expression level $(0.048)$ was found in patients with regression score $1(n=11)(p=0.044)$.

Analysis of the correlation between levels of miR-145 expression and dichotomized tumor regression in minor response (score 1 and 2) and major response (score 3 and 4) showed a trend torwards major response and higher miR-145 expression levels (Fig. 4).

Pre-therapeutic expression levels of miR-145 and correlation with tumor response. In view of tumor regression the highest level of pre-therapeutic miR-145 was found in grade 3 tumor regression followed by grades 2,1 and 4 . Analysis of the correlation between pre-therapeutic miR-145 expression and

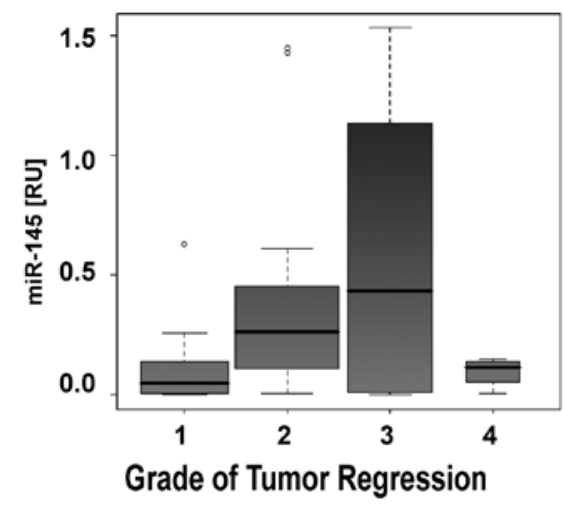

A.

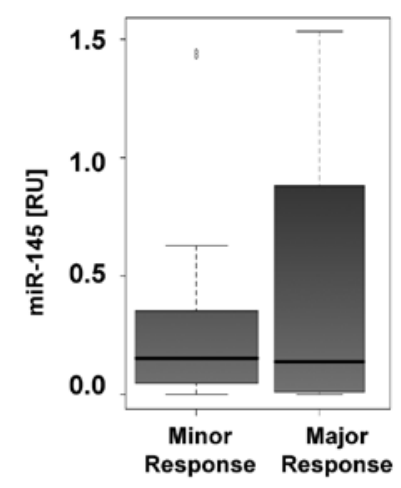

B.

Figure 4. Pathological tumor regression based on miR-145 expression in post-therapeutic tumor tissue. (A) Regression grades 1-4 (p=0.044). (B) Dichotomized regression in minor response (grades 1 and 2) and major response (grades 3 and 4) $(\mathrm{p}=0.631)$. 


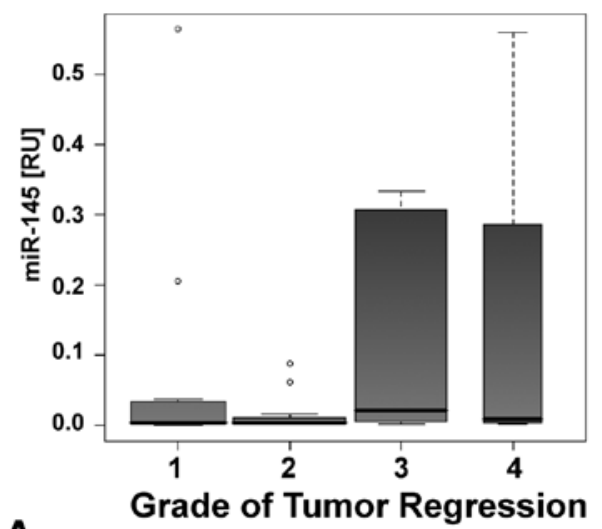

A.

Figure 5. Pathological tumor regression based on miR-145 expression in pre-therapeutic tumor tissue. (A) Regression grades 1-4 (p=0.201). (B) Dichotomized regression in minor response (grades 1 and 2) and major response (grades 3 and 4 ) at a cut-off value of 0.004 with the area under the ROC curve (AUC) of $0,696(\mathrm{p}=0.031)$.

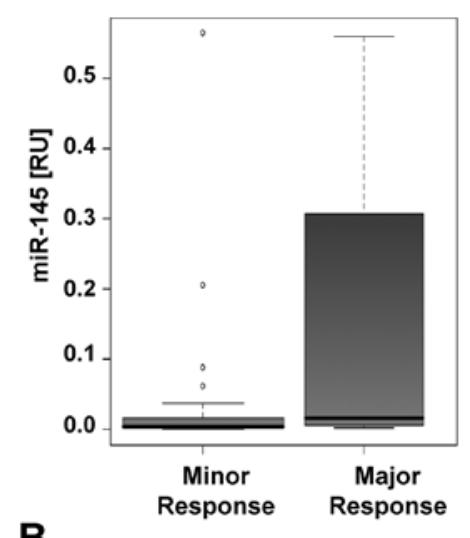

B.

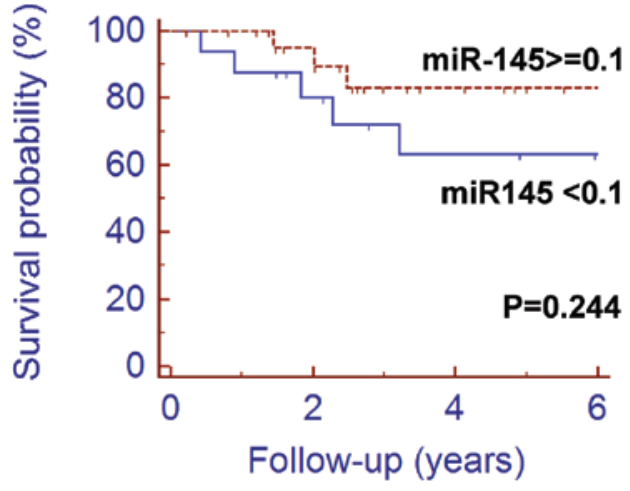

Figure 6. Kaplan-Meier overall survival curve based on dichotomized miR-145 expression in post-therapeutic tumor tissue at a cut-off value of 0.1 $(\mathrm{p}=0.244)$.

tumor response showed a correlation between major response and higher miR-145 expression at a cut-off value of 0.004 (Fig. 5).

Post-therapeutic miR-145 expression level and prognosis. Analysis of the correlation between prognosis and dichotomized post-therapeutic miR-145 expression showed a trend of longer survival of patients with miR-145 expression $>0.1 \quad(n=35)$ versus $<0.1(\mathrm{n}=15)$. This trend was not statistically significant $(\mathrm{p}=0.244)$ (Fig. 6).

\section{Discussion}

miRNAs seem to regulate many known oncogenic and tumor suppressor pathways involved in the pathogenesis of malignant tumors. Furthermore, expression profiles of hundreds of miRNAs have been shown to have at least the same potential for identification of biomarkers as profiling of their mRNA or protein counterparts (20).

In CRC, there is still a need to identify new prognostic markers and therapeutic targets. Overexpression and downregulation of specific miRNAs has been described in this disease $(20,23)$ and it has become evident that miRNAs are widely involved in the initiation and progression of CRC. According to this consideration, it is tempting to speculate that pharmacodynamic mechanisms of antineoplastic agents could rely on their influence on miRNA expression.

So far, there are only few studies addressing the question of the association of therapy response and miRNA expression (20). Nakajima et al identified let-7g and miR-181b as significant indicators for chemoresponse to 5-FU-based chemotherapy (26). Rossi et al reported a suggestive pattern of miRNA rearrangement in HT-29 and HCT-116 human colon cancer cell lines after exposure to 5-FU. In their study the drug upregulated or downregulated in vitro the expression of 19 and 3 miRNAs, respectively (23). Another study by Svoboda et al evaluated changes in miRNA expression profiles as a response to therapy by comparing pre-therapeutic tissue with biopsies taken two weeks after the beginning of capecitabine-based chemoradiotherapy (25). According to their results, many miRNAs responded to therapy, however only miR-125b and miR-137 showed significant increase in expression levels. Thus, more studies are needed to examine the effects of neoadjuvant chemoradiotherapy on miRNA expression, in order to analyse their putative role as biomarkers for prognosis and therapy response prediction in CRC.

In our study, we investigated the effect of neoadjuvant chemoradiotherapy with 5-FU and 50.4 Gy in cases of locally advanced rectal cancer on the expression levels of three different miRNAs, which are known to play critical roles to CRC. We investigated miR-21, which functions as an oncogene and miR-143 and miR-145 which were shown to act as tumor suppressors in CRC $(9,29)$, comparing expression levels of miRNAs in tumor tissue before and after treatment. The molecular mechanisms of 5-FU cytotoxicity have been characterized. 5-FU has the ability to incorporate into RNA and DNA and to inhibit thymidylate synthase. It has been shown that 5-FU modulates miRNA expression in colon cancer cells (23).

According to recent knowledge, miR-21 is expressed at high levels in most solid tumors, including CRC. Overexpression of miR-21 acts as an anti-apoptotic factor (10) and inhibition of miR-21 inhibits cell growth in vitro and inhibits tumor growth in xenograft mouse models through an indirect 
down-regulation of the anti-apoptotic factor B-cell lymphoma 2 (Bcl-2) (30). The tumor suppressor Pdcd4 is negatively regulated by miR-21 at the post-transcriptional level and miR-21 induces invasion/intra-vasation and metastasis in colorectal cancer cells $(21,31)$. miR-21 also acts as an activator of tumor cell proliferation (21). In our study, there was a trend towards higher expression levels of miR-21 in pre-therapeutic tumor tissue compared to post-therapeutic tumor tissue and normal adjacent tissue. Rossi et al illustrated for the first time that 5-FU induces changes of the miRNA expression profile in colon cancer cells. In their study, comparison of the miRNA gene expression revealed a rearrangement of a remarkable number of miRNAs after 5-FU treatment, and miRNA-21 is downregulated in colon cancer cell lines after treatment with 5-FU (23). Analogous to their results we also observed downregulation of miR-21 in the tumor tissue after treatment. Svoboda et al investigated the involvement of miRNAs in response to chemoradiotherapy comparing tumor biopsies of rectal cancer before and 2 weeks after starting anticancer treatment. In their study, they found for miR-21 in a pilot experiment of 6 patients a profound intertumoral variability and different exhibition of treatment responses (25). We could not find a statistically significant correlation between pre- and post-therapeutic miR-21 expression and response to treatment or prognosis. In contrast to our results, Schetter et al reported a significant correlation of high pre-therapeutic miR-21 expression levels and poor therapeutic outcome (32). However, they investigated adjuvant treated adenocarcinomas of the colon and investigated post-therapeutic tumor tissue and normal adjacent tissue which was collected after surgery.

miR-143 is known to be downregulated in colorectal cancer suggesting that it acts as a tumor-suppressor miRNA (15). Consistent with this, in our study we found that compared to post-therapeutic cancer tissue and normal adjacent tissue, miR-143 expression levels were significantly lower in pretherapeutic cancer tissue. Thus, miR-143 in response to chemoradiotherapy was upregulated. Nakajima et al investigated 46 colorectal adenocarcinomas and did not observe downregulation of miR-143 in tumor tissue compared to normal tissue. This controversy is discussed as a putative effect of genetic differences between Japanese and European patients (26). So far, little is known about the modification of miR-143 in the course of anticancer therapy. The relationship between 5-FU and miRNAs, their potential interactions and their relevance for drug efficacy have not been extensively studied. Borralho et al investigated the biological role of miR-143 in human colorectal cancer cells and found antiproliferative and proapoptotic roles for miR-143 in colon cancer. In addition to these findings, an increased sensitivity to 5-FU in human colorectal cancer cells was seen in association with an over-expression of miR-143 (24). In our study, the enhancing effect of the anticancer treatment by chemoradiotherapy on miR-143 expression seems to be in line with the antitumor properties of the treatment. Furthermore, we found a significant association between dichotomized post-therapeutic intratumoral miR-143 expression and survival probability.

In line with the tumor suppressive activity of the chemoradiotherapy of particular interest is our observation that also the expression level of miR-145 was upregulated in cancer tissue after treatment. Compared to normal tissue the pretherapeutic tumor tissue showed downregulation of miR-145 which is in line with Michael et al who described downregulation of miR-145 in CRC compared to normal tissue (15). In contrast to this finding, Nakajima et al could not find a decrease of miR-145 in CRC compared to normal tissue (26). However, the mechanism of post-therapeutic upregulation of miR-145 which we observed in our study remains elusive. To establish the direct link of miR-145 and chemotherapy response requires future studies in order to analyse the detailed molecular and cellular mechanisms. The association of miR-145 expression and grade of regression as well as survival probability strengthens the hypothesis of a putative role of miR-145 as a response-predicting and prognostic marker in the course of neoadjuvant treated CRC.

In conclusion, the results of this study confirm the findings of previous studies, that the use of neoadjuvant chemoradiotherapy can modify the expression profile of miRNAs in tumor tissue. The downregulation of the oncomir miR-21 and the upregulation of the tumor suppressor miR-143 and miR-145 in post-therapeutic tumor tissue stand in line with the antitumor properties of the therapy. The correlation of intratumoral post-therapeutic miR-143 expression and survival probability underlines the importance of miRNAs as prognostic biomarkers. The results could have significant clinical implications because they provide associations of miRNAs with neoadjuvant therapy, tumor regression and survival probability and strengthen the role of miRNAs as predictive molecular factors determining response to treatment and prognosis in CRC.

\section{Acknowledgements}

We greatly appreciate the technical assistance of Desiree Kaptein, Melanie Scheffler and Hannah Eischeid.

\section{References}

1. Schmiegel W, Pox C, Reinacher-Schick A, Adler G, Arnold D, Fleig W, Folsch UR, Fruhmorgen P, Graeven U, Heinemann V, Hohenberger W, Holstege A, Junginger T, Kopp I, Kuhlbacher T, Porschen R, Propping P, Riemann JF, Rodel C, Sauer R, Sauerbruch T, Schmitt W, Schmoll HJ, Seufferlein T, Zeitz M and Selbmann HK: S3 guidelines for colorectal carcinoma: results of an evidence-based consensus conference on February 6/7, 2004 and June 8/9, 2007 (for the topics IV, VI and VII). Z Gastroenterol 48: 65-136, 2010.

2. Bosset JF, Calais G, Daban A, Berger C, Radosevic-Jelic L, Maingon P, Bardet E, Pierart M and Briffaux A: Preoperative chemoradiotherapy versus preoperative radiotherapy in rectal cancer patients: assessment of acute toxicity and treatment compliance. Report of the 22921 randomised trial conducted by the EORTC Radiotherapy Group. Eur J Cancer 40: 219-224, 2004.

3. Rodel C, Martus P, Papadoupolos T, Fuzesi L, Klimpfinger M, Fietkau R, Liersch T, Hohenberger W, Raab R, Sauer R and Wittekind C: Prognostic significance of tumor regression after preoperative chemoradiotherapy for rectal cancer. J Clin Oncol 23: 8688-8696, 2005.

4. Rimkus C, Friederichs J, Boulesteix AL, Theisen J, Mages J, Becker K, Nekarda H, Rosenberg R, Janssen KP and Siewert JR: Microarray-based prediction of tumor response to neoadjuvant radiochemotherapy of patients with locally advanced rectal cancer. Clin Gastroenterol Hepatol 6: 53-61, 2008.

5. Berezikov E, Guryev V, van de Belt J, Wienholds E, Plasterk RH and Cuppen E: Phylogenetic shadowing and computational identification of human microRNA genes. Cell 120: 21-24, 2005. 
6. Bentwich I, Avniel A, Karov Y, Aharonov R, Gilad S, Barad O, Barzilai A, Einat P, Einav U, Meiri E, Sharon E, Spector Y and Bentwich Z: Identification of hundreds of conserved and nonconserved human microRNAs. Nat Genet 37: 766-770, 2005.

7. Krutzfeldt J, Rajewsky N, Braich R, Rajeev KG, Tuschl T, Manoharan M and Stoffel M: Silencing of microRNAs in vivo with 'antagomirs'. Nature 438: 685-689, 2005.

8. Mendell JT: microRNAs: critical regulators of development, cellular physiology and malignancy. Cell Cycle 4: 1179-1184, 2005.

9. Shenouda SK and Alahari SK: microRNA function in cancer: oncogene or a tumor suppressor? Cancer Metastasis Rev 28: 369-378, 2009.

10. Chen X, Guo X, Zhang H, Xiang Y, Chen J, Yin Y, Cai X, Wang K, Wang G, Ba Y, Zhu L, Wang J, Yang R, Zhang Y, Ren Z, Zen K, Zhang J and Zhang CY: Role of miR-143 targeting KRAS in colorectal tumorigenesis. Oncogene 28: 1385-1392, 2009.

11. Calin GA and Croce CM: microRNA signatures in human cancers. Nat Rev Cancer 6: 857-866, 2006.

12. Esquela-Kerscher A and Slack FJ: Oncomirs-microRNAs with a role in cancer. Nat Rev Cancer 6: 259-269, 2006.

13. Lim LP, Lau NC, Garrett-Engele P, Grimson A, Schelter JM, Castle J, Bartel DP, Linsley PS and Johnson JM: Microarray analysis shows that some microRNAs downregulate large numbers of target mRNAs. Nature 433: 769-773, 2005.

14. Slaby O, Svoboda M, Fabian P, Smerdova T, Knoflickova D Bednarikova M, Nenutil R and Vyzula R: Altered expression of miR-21, miR-31, miR-143 and miR-145 is related to clinicopathologic features of colorectal cancer. Oncology 72: 397-402, 2007.

15. Michael MZ, SM OC, van Holst Pellekaan NG, Young GP and James RJ: Reduced accumulation of specific microRNAs in colorectal neoplasia. Mol Cancer Res 1: 882-891, 2003.

16. Akao Y, Nakagawa Y and Naoe T: MicroRNAs 143 and 145 are possible common onco-microRNAs in human cancers. Oncol Rep 16: 845-850, 2006.

17. Bandres E, Cubedo E, Agirre X, Malumbres R, Zarate R Ramirez N, Abajo A, Navarro A, Moreno I, Monzo M and Garcia-Foncillas J: Identification by real-time PCR of 13 mature microRNAs differentially expressed in colorectal cancer and non-tumoral tissues. Mol Cancer 5: 29, 2006.

18. Iorio MV, Ferracin M, Liu CG, Veronese A, Spizzo R, Sabbioni S, Magri E, Pedriali M, Fabbri M, Campiglio M, Menard S, Palazzo JP, Rosenberg A, Musiani P, Volinia S, Nenci I, Calin GA, Querzoli P, Negrini $M$ and Croce CM: microRNA gene expression deregulation in human breast cancer. Cancer Res 65: 7065-7070, 2005 .

19. La Rocca G, Badin M, Shi B, Xu SQ, Deangelis T, SeppLorenzinoi L and Baserga R: Mechanism of growth inhibition by MicroRNA 145: the role of the IGF-I receptor signaling pathway. J Cell Physiol 220: 485-491, 2009.
20. Slaby O, Svoboda M, Michalek $\mathbf{J}$ and Vyzula R: microRNAs in colorectal cancer: translation of molecular biology into clinical application. Mol Cancer 8: 102, 2009.

21. Asangani IA, Rasheed SA, Nikolova DA, Leupold JH, Colburn NH, Post S and Allgayer H: microRNA-21 (miR-21) post-transcriptionally downregulates tumor suppressor Pdcd4 and stimulates invasion, intravasation and metastasis in colorectal cancer. Oncogene 27: 2128-2136, 2008.

22. Allgayer H: Pdcd4, a colon cancer prognostic that is regulated by a microRNA. Crit Rev Oncol Hematol 73: 185-191, 2010.

23. Rossi L, Bonmassar E and Faraoni I: Modification of miR gene expression pattern in human colon cancer cells following exposure to 5-fluorouracil in vitro. Pharmacol Res 56: 248-253, 2007.

24. Borralho PM, Kren BT, Castro RE, da Silva IB, Steer CJ and Rodrigues CM: microRNA-143 reduces viability and increases sensitivity to 5-fluorouracil in HCT116 human colorectal cancer cells. FEBS J 276: 6689-6700, 2009.

25. Svoboda M, Izakovicova Holla L, Sefr R, Vrtkova I, Kocakova I, Tichy B and Dvorak J: Micro-RNAs miR125b and miR137 are frequently upregulated in response to capecitabine chemoradiotherapy of rectal cancer. Int J Oncol 33: 541-547, 2008.

26. Nakajima G, Hayashi K, Xi Y, Kudo K, Uchida K, Takasaki K, Yamamoto $\mathrm{M}$ and $\mathrm{Ju} \mathrm{J}$ : Non-coding microRNAs hsa-let-7g and hsa-miR-181b are associated with chemoresponse to S-1 in colon cancer. Cancer Genomics Proteomics 3: 317-324, 2006.

27. Schneider PM, Baldus SE, Metzger R, Kocher M, Bongartz R, Bollschweiler E, Schaefer H, Thiele J, Dienes HP, Mueller RP and Hoelscher AH: Histomorphologic tumor regression and lymph node metastases determine prognosis following neoadjuvant radiochemotherapy for esophageal cancer: implications for response classification. Ann Surg 242: 684-692, 2005.

28. Varnholt H, Drebber U, Schulze F, Wedemeyer I, Schirmacher P, Dienes HP and Odenthal M: MicroRNA gene expression profile of hepatitis C virus-associated hepatocellular carcinoma. Hepatology 47: 1223-1232, 2008.

29. Zhang B, Pan X, Cobb GP and Anderson TA: microRNAs as oncogenes and tumor suppressors. Dev Biol 302: 1-12, 2007.

30. Si ML, Zhu S, Wu H, Lu Z, Wu F and Mo YY: miR-21-mediated tumor growth. Oncogene 26: 2799-2803, 2007.

31. Chen Y, Liu W, Chao T, Zhang Y, Yan X, Gong Y, Qiang B, Yuan J, Sun M and Peng X: MicroRNA-21 down-regulates the expression of tumor suppressor PDCD4 in human glioblastoma cell T98G. Cancer Lett 272: 197-205, 2008

32. Schetter AJ, Leung SY, Sohn JJ, Zanetti KA, Bowman ED, Yanaihara N, Yuen ST, Chan TL, Kwong DL, Au GK, Liu CG, Calin GA, Croce CM and Harris CC: microRNA expression profiles associated with prognosis and therapeutic outcome in colon adenocarcinoma. JAMA 299: 425-436, 2008. 